\title{
Neues aus der Deutschen Initiative für Netzwerkinformation (DINI): Online-Jahrestagung und Veröffentlichungen
}

\author{
Die Deutschen Initiative für Netzwerkinformation (DINI) \\ informiert über ihre Online-Jahrestagung sowie über mehrere \\ Publikationen rund um die Digitalisierung von Forschung und \\ Lehre
}

https://doi.org/10.1515/bd-2020-0080

\section{Call for Papers: DINI-Jahrestagung 2020}

Aufgrund der Corona-Pandemie wird die 21. Jahrestagung der Deutschen Initiative für Netzwerkinformation (DINI) nicht wie geplant in Nürnberg, sondern online stattfinden.

DINI nimmt dies zum Anlass, sich mit den Herausforderungen der Pandemie für Dienstleistungseinrichtungen in Forschung und Lehre zu beschäftigen.

Im Fokus stehen die Herausforderungen, denen sich Dienstleistungseinrichtungen im Wandel der Hochschulen und Forschungseinrichtungen unterworfen sehen. Aufbau und Nutzung der Serviceportfolios in den DINI-Mitgliedseinrichtungen haben 2020 durch die COVID-bedingten Kontaktbeschränkungen eine bis dato unbekannte Dynamik entfaltet: Zahlreiche Veranstaltungen und Arbeitsroutinen mussten auf digital gestützte Formate umgestellt werden.

Die DINI-Jahrestagung am 29. und 30. September 2020 bietet Gelegenheit für Austausch und Reflexion dieser Entwicklungen - unter digitalen Bedingungen -, denn die Jahrestagung wird online stattfinden.

Passend zum Leitthema der Tagung - User Experience - soll dabei der Fokus direkt auf Erfahrungen liegen, die im Zuge der „Corona-Lehre“ und des Arbeitens unter digitalen Vorzeichen gewonnen werden.

Angesprochen sind Nutzergruppen von Studierenden, über Lehrende bis hin zu Verantwortlichen in Bibliotheken, Medien- und Rechenzentren und Verwaltung.

Auf der Jahrestagung stellt DINI deren Erfahrungen in den Mittelpunkt. Welche digitalen Entwicklungen sind ihre Best Practices? Welche organisatorische und welche technischen Rahmenbedingungen haben sie als wichtig für Forschung, Lehre und Lernen kennengelernt? DINI ruft zu Beiträgen aus der Community auf. Das Organisationskomitee wird anhand der Einreichungen über die Gestaltung der Sessions entscheiden. Die Einreichung ist bis zum 27.07.2020 möglich. 
Weiterführende Informationen: https://dini.de/veranstaltungen/jahrestagun gen/21-dini-jahrestagung-2020.

\section{Neue Publikation: „Nachhaltige Kooperationsstrukturen zur Unterstützung der Digitalisierung an Hochschulen“}

Die DINI-Arbeitsgruppe/ZKI-Kommission „E-Framework“ legt die Publikation „Nachhaltige Kooperationsstrukturen zur Unterstützung der Digitalisierung an Hochschulen“ vor.

Die Anforderungen an die Leistungsfähigkeit und Verfügbarkeit der Serviceportfolios zur nachhaltigen Unterstützung der Digitalisierung in Forschung, Lehre, Studium und Verwaltung steigen kontinuierlich. Angesichts dessen werden auf lokaler und regionaler Ebene Service-Infrastrukturen in Form von Kooperationen zwischen den jeweils verantwortlichen Einrichtungen aufgebaut. Im Hinblick auf die Nachnutzbarkeit, Nachhaltigkeit und Kosteneffizienz lokal oder regional verfügbarer Service-Infrastrukturen entsteht zunehmend der Bedarf an regionalen, länderübergreifenden und nationalen Kooperationsstrukturen. Damit stellt sich die Frage, mit welchen Organisations- und Rechtsformen länderübergreifend bzw. national verfügbare Servicestrukturen dauerhaft betrieben und genutzt werden können: in Form von Verträgen zwischen Hochschulen, als Genossenschaftsmodell, gemeinnützige GmbHs, per Vereinsgründung oder Verwaltungsabkommen etc. Dazu stellen sich viele Fragen, die in der vorliegenden Veröffentlichung aufgegriffen werden.

Als Einstieg in die Thematik hat die DINI-Arbeitsgruppe/ZKI-Kommission „E-Framework“ im Dezember 2018 an der Universität Hamburg einen Workshop durchgeführt, auf dem eine Reihe von Beispielen für Kooperationsmodelle vorgestellt wurden, die erfolgreich auf nationaler Ebene realisiert wurden. Die Ergebnisse des Workshops werden in der Veröffentlichung vorgestellt. Das Papier wendet sich sowohl an Ministerien, Hochschulleitungen und Leitungen zentraler Einrichtungen als auch an Projektleitungen, um wesentliche Aspekte erfolgreicher und nachhaltiger Kooperationsmodelle zur Serviceerbringung aufzuzeigen und um auf diese Weise zur Entscheidungsfindung beizutragen.

Die Publikation wurde Open Access veröffentlicht: https://doi.org/10.18452/ 19999.

\section{Save the Date: Fünf Jahre Kerndatensatz Forschung im deutschen Wissen- schaftssystem - Umsetzung, Entwicklungen und Perspektiven}

Am 9. und 10. November 2020 organisieren das Deutsche Zentrum für Hochschulund Wissenschaftsforschung und die DINI-Arbeitsgruppe „Forschungsinformationssysteme“ eine öffentlich zugängliche virtuelle Veranstaltung zum Thema Kerndatensatz Forschung. 
Mit der Veranstaltung werden nunmehr fünf Jahre Kerndatensatz Forschung zum Anlass genommen, um über dessen Umsetzung, dabei gemachte Erfahrungen sowie Entwicklungen und Perspektiven zu sprechen und sich auszutauschen. Damit richtet sich die Veranstaltung an Nutzerinnen und Nutzer von Forschungsinformationen und Forschungsinformationssystemen, Beauftragte zur Umsetzung des Kerndatensatzes Forschung, Forschungsreferentinnen und -referenten, Verantwortliche in Bibliotheken und Rechenzentren sowie Vertreterinnen und Vertreter von Bundes- und Landesministerien und Forschungsförderern.

Die Veranstaltung beschäftigt sich mit dem Stand und den Prozessen der Einführung im deutschen Wissenschaftssystem, beleuchtet aktuelle Entwicklungen rund um die Erhebung und Verarbeitung von Daten, bietet Raum zur Diskussion von Chancen und Grenzen der Nutzbarkeit des Kerndatensatzes Forschung für bestehende Informationsbedarfe und Berichtszwecke und setzt sich mit zukünftigen Entwicklungen rund um den Standard auseinander.

Weitere Informationen: https://dini.de/veranstaltungen/workshops/fuenfjahre-kerndatensatz-forschung-im-deutschen-wissenschaftssystem-umsetzungentwicklungen-und-perspektiven.

\section{Aktualisierung der Liste der Open Access-Publikationsdienste in Deutsch- land}

Die DINI-Arbeitsgruppe „Elektronisches Publizieren“ (E-Pub) bietet gemeinsam mit BASE (Bielefeld Academic Search Engine) eine umfangreiche Liste aller Open Access-Publikationsdienste in Deutschland an. Die automatisiert generierte Liste zeigt u.a. den Anteil von Open Access-Publikationen, vorhandene Zertifikate und lässt sich nach Typ des Publikationsdienstes, genutzter Software-Plattform und Region filtern.

Durch die Kooperation mit BASE werden Synergien bei der Bereitstellung von Informationen zu Open Access-Publikationen nachhaltig und ressourcenschonend umgesetzt.

Weitere Informationen: https://dini.de/dienste-projekte/publikationsdienste.

\section{„Zukunft Lernwelt Hochschule“ frei verfügbar}

An die Lernwelt Hochschule werden neue Anforderungen gestellt. Die Veränderungen im Rahmen des Bologna-Prozesses sowie die zunehmende Digitalisierung haben gravierende Auswirkungen auf die Universitäten und Hochschulen. Die Einführung von aktivierenden, team- und projektorientierten Lehrformaten lassen Orte und Angebote zum Selbststudium immer wichtiger werden. Im Forschungsprojekt Lernwelt Hochschule (LeHo) wurde im Zeitraum 2017-2019 der Frage nachgegangen, wie die zukünftige Gestaltung von physischen, digitalen 
und hybriden Lernräumen an den Hochschulen aussieht. Projektpartner sind die Hochschule der Medien Stuttgart (HdM) in Zusammenarbeit mit der Hochschule für Angewandte Wissenschaften Hamburg, der Otto-Friedrich-Universität Bamberg, der Heinrich-Heine-Universität Düsseldorf und DINI. Finanziert wurde das Projekt von der Dieter Schwarz Stiftung.

Weiterführende Informationen: http://leho.blog und https://zukunftlern welthochschule.de.

Aus dem Projekt gehen zwei Buchpublikationen hervor. Band 1 „Lernwelt Hochschule. Dimensionen eines Bildungsbereichs im Umbruch“ beschreibt den State-of-the-art mit Herausforderungen und Good-Practice-Beispielen. Band 2 „Zukunft Lernwelt Hochschule. Perspektiven und Optionen für eine Neuausrichtung“ richtet einen Blick von außen auf die Entwicklungen der Lernwelt Hochschule und entwirft einen Baukasten zur Entwicklung von Good-PracticeBeispielen. Beide Bände sind bei De Gruyter auch im Open Access erschienen: https://www.degruyter.com/view/title/536818 und https://www.degruyter.com/ view/title/552482.

Um die Forschungsergebnisse zu visualisieren, wurde auf Wiki-Basis ein DINI Lernraum-Atlas erstellt: https://intern.dini.de/confluence/display/LEHO (im Juli 2020 frei zugänglich). Neben der Beschreibung der vier Dimensionen „Hochschulorganisation“, „Digitale Strukturen“, „Hochschuldidaktik“ und „Physische Lehrund Lernräume“ stellen sich auch die im Rahmen des Projekts interviewten Hochschulen bzw. Hochschulen, die sich an der Online-Umfrage beteiligt haben, vor. Gerne können Informationen angepasst werden und neue Hochschulen ergänzt werden.

\section{Neue Ausgabe der „Bausteine Forschungsdatenmanagement“}

Die von der DINI/nestor Arbeitsgruppe „Forschungsdaten“ herausgegebene Open Access-Zeitschrift „Bausteine Forschungsdatenmanagement“ (https://bausteinefdm.de) richtet sich an Praktikerinnen und Praktiker im Bereich des Forschungsdatenmanagements (FDM). „Bausteine Forschungsdatenmanagement“ dient dem Austausch von Erfahrungen und von daraus abgeleiteten praktischen Handlungsempfehlungen.

In der aktuellen Ausgabe (Heft 1/2020) finden sich neben Artikeln zur „FDM-Kompetenzausbildung“ und zu „Kosten und Aufwänden des FDM“ zahlreiche Beiträge, die im Rahmen der Workshopreihe „FDM am Standort: von der initialen Idee zum dauerhaften Service“ entstanden sind, die von der Arbeitsgruppe Forschungsdaten zwischen Januar und April 2020 organisiert wurde.

Auch der Sonderband mit Beiträgen des Workshops „Forschungsdatenmanagement und -infrastruktur in DFG-Sonderforschungsbereichen“ wurde mit Veröffentlichung von Heft 1/2020 um weitere Artikel ergänzt. 
Die Redaktion freut sich jederzeit über die Einreichung von Beiträgen aus dem Themenfeld „Praxis des Forschungsdatenmanagements“.

\section{Jubiläum und Premiere: die 10. Auflage des KIM-Workshops}

Der jährliche Workshop der DINI-Arbeitsgruppe „Kompetenzzentrum Interoperable Metadaten“ (KIM) feierte Anfang Mai nicht nur 10. Jubiläum, sondern auch erfolgreiche Online-Premiere. Dabei konnte neben klassischen Fachvorträgen auch der freie Community-Austausch - etwas reduziert, aber lebendig - stattfinden, etwa in Form von Lightning Talks und einem Open Space-Slot.

Mit phasenweise über hundert Teilnehmerinnen und Teilnehmern konnten mehr Interessierte als gewöhnlich begrüßt werden, was sicherlich auch an dem niedrigschwelligen Online-Format lag.

Die Universitätsbibliothek Mannheim blieb auch im 10. Workshop-Jahr Gastgeberin der Veranstaltung, indem sie die Online-Plattform zur Verfügung stellte.

Alle Folien zu u.a. den Themen Metadaten-Anwendungsprofile, Metadaten im Bibliotheksmanagementsystem FOLIO, kooperative Pflege von Spezifikationen und der Software Wikibase finden sich unter: https://wiki.dnb.de/x/a_A8CQ.

\section{Über die Deutsche Initiative für Netzwerkinformation (DINI)}

Die Deutsche Initiative für Netzwerkinformation (DINI) e. V. ist der überregionale Zusammenschluss von wissenschaftlichen Bibliotheken, Medienzentren, Rechenzentren und Fachgesellschaften in Forschung und Lehre in Deutschland. DINI ist Partnerin von Hochschulen und außeruniversitären Forschungseinrichtungen bei der Weiterentwicklung der Informationsinfrastrukturen.

Weitere Informationen: https://dini.de. 